\title{
NN scattering formulations without partial-wave decomposition
}

\author{
Imam Fachruddin* \\ Departemen Fisika, Universitas Indonesia, Depok 16424, Indonesia \\ E-mail: imam.fachruddinesci.ui.ac.id
}

\section{Agus Salam}

Departemen Fisika, Universitas Indonesia, Depok 16424, Indonesia

\begin{abstract}
Recently a new three-dimensional (3D) formulation for scattering of two spin-half particles, either identical or unidentical, is presented. In the formulation the free linear-momentum states are not expanded into the angular-momentum states and the system's spin and isospin states, respectively, are described by the product of the spin and isospin states of the two particles. We apply this technique to calculate nucleon-nucleon (NN) scattering. But as NN interaction models are usually being parametrized regarding to the $\mathrm{NN}$ total isospin, we take the total isospin state, instead of the product of the isospin states of the two nucleons, as part of the $3 \mathrm{D}$ basis state. We evaluate the Lippmann-Schwinger equations for NN T-matrix elements in these 3D basis states and end up with a set of coupled integral equations for the NN T-matrix elements in two variables, namely the magnitude of the relative momentum and the scattering angle. We show as an example the spinaveraged differential cross section, which can be calculated directly from the solution of the set of the integral equations. The recently developed $3 \mathrm{D}$ formulation also introduces a set of six spin operators to express any interaction of two spin-half particles. We show the Bonn NN potential in terms of these six spin operators.
\end{abstract}

XV International Conference on Hadron Spectroscopy

4-8 November 2013

Nara, Japan

\footnotetext{
* Speaker.
} 


\section{Introduction}

The fact that nucleon-nucleon $(\mathrm{NN})$ interaction is of short-range interaction allows one to analyze NN scattering by considering only a number of lowest angular-momentum states or partial waves. But as the energy at which the scattering occurs increases many more higher partial waves are needed[1,2]. For energies up to a few hundreds $\mathrm{MeV}$ one faces no significant difficulties to perform well-converging partial-wave (PW) calculations for NN scattering, but not for threenucleon $(3 \mathrm{~N})$ scattering[3].

There are some works to formulate and calculate $\mathrm{NN}$ and $3 \mathrm{~N}$ scattering in three dimensions without PW decomposition, see, for example, Refs. [1]-[7], and they give good results. Here we develope a new three-dimensional (3D) formulation for NN scattering, which is based on our previous work in Ref. [8]. Although 3D calculations are required more for 3N scattering, we start from NN scattering, since the NN T-matrix elements are input to calculations of $3 \mathrm{~N}$ scattering.

\section{NN scattering in new three-dimensional formulation}

Let us take a NN observable, for example the spin-averaged differential cross section:

$$
\frac{\overline{d \sigma}}{d \hat{\mathbf{p}}^{\prime}}=\frac{1}{4} \mu^{2}(2 \pi)^{4} \sum_{\lambda_{1}^{\prime}, \lambda_{2}^{\prime}, \lambda_{1}, \lambda_{2}=-\frac{1}{2}}^{\frac{1}{2}}\left|\sum_{\tau=0}^{1} C^{2}\left(\frac{1}{2} \frac{1}{2} \tau ; v_{1} v_{2} v\right)\left\langle p \hat{\mathbf{p}}^{\prime} \lambda_{1}^{\prime} \lambda_{2}^{\prime} \tau \nu\left|T^{a}\right| \mathbf{p} \lambda_{1} \lambda_{2} \tau v\right\rangle\right|^{2}
$$

with

$$
\left|\mathbf{p} \lambda_{1} \lambda_{2} \tau v\right\rangle \equiv|\mathbf{p}\rangle\left|\lambda_{1}\right\rangle\left|\lambda_{2}\right\rangle|\tau v\rangle
$$

Here $T^{a} \equiv\left(1-P_{12}\right) T$ is the antisymmetrized NN T-matrix, $P_{12}$ a permutation operator, $\mathbf{p}$ the relative momentum, $\left|\lambda_{i}\right\rangle(i=1,2)$ the spin state of the $i^{\text {th }}$ nucleon, with the spin being quantized along the $\mathrm{z}$ axis, $\tau$ and $v$ respectively the $\mathrm{NN}$ total isospin quantum number and the quantum number for the z-component of the NN total isospin, $\mu$ the reduction mass, and $C\left(\frac{1}{2} \frac{1}{2} \tau ; v_{1} v_{2} v\right)$ ClebschGordan coefficients. So, to calculate observables one needs $\left\langle p \hat{\mathbf{p}}^{\prime} \lambda_{1}^{\prime} \lambda_{2}^{\prime} \tau \nu\left|T^{a}\right| \mathbf{p} \lambda_{1} \lambda_{2} \tau \nu\right\rangle$. Hence, in our 3D formulation we derive the Lippmann-Schwinger (LS) equation for the T-matrix elements $\left\langle p \hat{\mathbf{p}}^{\prime} \lambda_{1}^{\prime} \lambda_{2}^{\prime} \tau \nu\left|T^{a}\right| \mathbf{p} \lambda_{1} \lambda_{2} \tau \nu\right\rangle$. This means we take the state given in Eq. (2.2) as basis state.

Different from the 3D basis state in Ref. [8], here we take the NN total isospin state $|\tau v\rangle$ instead of the product of the isospin states of the two nucleons $\left|v_{1}\right\rangle\left|v_{2}\right\rangle$. We do this, since models of NN potential are usually parametrized with respect to the total isospin of the NN system.

We evaluate in the 3D basis states $\left|\mathbf{p} \lambda_{1} \lambda_{2} \tau v\right\rangle$ the following LS equation for the antisymmetrized T-matrix $T^{a}$, with $V^{a} \equiv\left(1-P_{12}\right) V$ :

$$
T^{a}=V^{a}+V G_{0} T^{a}
$$

We obtain

$$
T_{\lambda_{1}^{\prime} \lambda_{2}^{\prime} \lambda_{1} \lambda_{2}}^{a, \tau v}\left(\mathbf{p}^{\prime}, \mathbf{p}\right)=V_{\lambda_{1}^{\prime} \lambda_{2}^{\prime} \lambda_{1} \lambda_{2}}^{a, \tau \nu}\left(\mathbf{p}^{\prime}, \mathbf{p}\right)+\sum_{\lambda_{1}^{\prime \prime}, \lambda_{2}^{\prime \prime}=-\frac{1}{2}}^{\frac{1}{2}} \int d \mathbf{p}^{\prime \prime} V_{\lambda_{1}^{\prime} \lambda_{2}^{\prime} \lambda_{1}^{\prime \prime} \lambda_{2}^{\prime \prime}}^{\tau \nu}\left(\mathbf{p}^{\prime}, \mathbf{p}^{\prime \prime}\right) G_{0}^{+}\left(p^{2}\right) T_{\lambda_{1}^{\prime \prime} \lambda_{2}^{\prime \prime} \lambda_{1} \lambda_{2}}^{a, \tau v}\left(\mathbf{p}^{\prime \prime}, \mathbf{p}\right),
$$


with $G_{0}^{+}\left(p^{2}\right)=\lim _{\varepsilon \rightarrow 0} \frac{2 \mu}{p^{2}+i \varepsilon-p^{\prime 2}}$ and the potential matrix elements (similarly the T-matrix elements) being defined as

$$
\begin{aligned}
& V_{\lambda_{1}^{\prime} \lambda_{2}^{\prime} \lambda_{1} \lambda_{2}}\left(\mathbf{p}^{\prime}, \mathbf{p}\right) \equiv\left\langle\mathbf{p}^{\prime} \lambda_{1}^{\prime} \lambda_{2}^{\prime} \tau v|V| \mathbf{p} \lambda_{1} \lambda_{2} \tau v\right\rangle \\
& V_{\lambda_{1}^{a}, \tau v} \lambda_{2}^{\prime} \lambda_{1} \lambda_{2} \\
& \left.\mathbf{p}^{\prime}, \mathbf{p}\right) \equiv\left\langle\mathbf{p}^{\prime} \lambda_{1}^{\prime} \lambda_{2}^{\prime} \tau v\left|V^{a}\right| \mathbf{p} \lambda_{1} \lambda_{2} \tau \nu\right\rangle .
\end{aligned}
$$

As for $\hat{\mathbf{p}}=\hat{\mathbf{z}}$ the azimuthal dependency of the potential matrix elements (and similarly of the Tmatrix elements) shows up as a phase factor as

$$
\begin{aligned}
& V_{\lambda_{1}^{\prime} \lambda_{2}^{\prime} \lambda_{1} \lambda_{2}}^{\tau\left(\mathbf{p}^{\prime}, p \hat{\mathbf{z}}\right)}=e^{-i\left(\lambda_{1}^{\prime}+\lambda_{2}^{\prime}-\lambda_{1}-\lambda_{2}\right) \phi^{\prime}} V_{\lambda_{1}^{\prime} \lambda_{2}^{\prime} \lambda_{1} \lambda_{2}}^{\tau v}\left(p^{\prime}, p, \theta^{\prime}\right) \\
& V_{\lambda_{1}^{\prime} \lambda_{2}^{\prime} \tau v}^{\prime \tau} \lambda_{1} \lambda_{2} \\
& \left(\mathbf{p}^{\prime}, p \hat{\mathbf{z}}\right)=e^{-i\left(\lambda_{1}^{\prime}+\lambda_{2}^{\prime}-\lambda_{1}-\lambda_{2}\right) \phi^{\prime}} V_{\lambda_{1}^{\prime} \lambda_{2}^{\prime} \lambda_{1} \lambda_{2}}^{(\tau v}\left(p^{\prime}, p, \theta^{\prime}\right),
\end{aligned}
$$

the LS equation for $T_{\lambda_{1}^{\prime} \lambda_{2}^{\prime} \lambda_{1} \lambda_{2}}^{a}\left(\mathbf{p}^{\prime}, \mathbf{p}\right)$ given in Eq. (2.4) finally reduces to the following integral equation for $T_{\lambda_{1}^{\prime} \lambda_{2}^{\prime} \lambda_{1} \lambda_{2}}^{a, v}\left(p^{\prime}, p, \theta^{\prime}\right)$ :

$$
\begin{aligned}
T_{\lambda_{1}^{\prime} \lambda_{2}^{\prime} \lambda_{1} \lambda_{2}}^{a, \tau}\left(p^{\prime}, p, \theta^{\prime}\right)=V_{\lambda_{1}^{\prime} \lambda_{2}^{\prime} \lambda_{1} \lambda_{2}}^{a, \tau v} & \left(p^{\prime}, p, \theta^{\prime}\right)+2 \mu \lim _{\varepsilon \rightarrow 0} \sum_{\lambda_{1}^{\prime \prime}, \lambda_{2}^{\prime \prime}=-\frac{1}{2}}^{\frac{1}{2}} \int_{0}^{\infty} d p^{\prime \prime} \frac{p^{\prime \prime 2}}{p^{2}+i \varepsilon-p^{\prime \prime 2}} \\
& \times \int_{-1}^{1} d \cos \theta^{\prime \prime} V_{\lambda_{1}^{\prime} \lambda_{2}^{\prime} \lambda_{1}^{\prime \prime} \lambda_{2}^{\prime \prime}}^{\tau v\left(\lambda_{2} \lambda_{2}\right)}\left(p^{\prime}, p^{\prime \prime}, \theta^{\prime}, \theta^{\prime \prime}\right) T_{\lambda_{1}^{\prime \prime} \lambda_{2}^{\prime \prime} \lambda_{1} \lambda_{2}}^{a, \tau \nu}\left(p^{\prime \prime}, p, \theta^{\prime \prime}\right),
\end{aligned}
$$

with

$$
V_{\lambda_{1}^{\prime} \lambda_{2}^{\prime} \lambda_{1}^{\prime \prime} \lambda_{2}^{\prime \prime}}^{\tau \nu,\left(\lambda_{2}+\lambda_{2}\right)}\left(p^{\prime}, p^{\prime \prime}, \theta^{\prime}, \theta^{\prime \prime}\right) \equiv \int_{0}^{2 \pi} d \phi^{\prime \prime} V_{\lambda_{1}^{\prime} \lambda_{2}^{\prime} \lambda_{1}^{\prime \prime} \lambda_{2}^{\prime \prime}}^{\tau v}\left(\mathbf{p}^{\prime}, \mathbf{p}^{\prime \prime}\right) e^{i\left\{\left(\lambda_{1}^{\prime}+\lambda_{2}^{\prime}\right) \phi^{\prime}-\left(\lambda_{1}^{\prime \prime}+\lambda_{2}^{\prime \prime}\right) \phi^{\prime \prime}\right\}} e^{-i\left(\lambda_{1}+\lambda_{2}\right)\left(\phi^{\prime}-\phi^{\prime \prime}\right)} .
$$

For a system of two spin-half particles it is known that symmetry behaviour of the T-matrix elements reduces the number of T-matrix elements to be taken, with respect to various initial and final spin states, from 16 to 6 . We get this symmetry behaviour for $T_{\lambda_{1}^{\prime} \lambda_{2}^{\prime} \lambda_{1} \lambda_{2}}^{a, \tau \nu}\left(p^{\prime}, p, \theta^{\prime}\right)$ as

$$
\begin{aligned}
& T_{\lambda_{1}^{\prime} \lambda_{2}^{\prime} \lambda_{1} \lambda_{2}}^{a, \tau v}\left(p^{\prime}, p, \theta^{\prime}\right)=(-)^{\lambda_{1}^{\prime}+\lambda_{2}^{\prime}+\lambda_{1}+\lambda_{2}} T_{-\lambda_{1}^{\prime},-\lambda_{2}^{\prime},-\lambda_{1},-\lambda_{2}}^{a, \tau v}\left(p^{\prime}, p, \theta^{\prime}\right) \\
& T_{\lambda_{1}^{a} \lambda_{2}^{\prime} \lambda_{1} \lambda_{2}}^{a}\left(p^{\prime}, p, \theta^{\prime}\right)=T_{\lambda_{2}^{\prime} \lambda_{1}^{\prime} \lambda_{2} \lambda_{1}}^{a, \tau v}\left(p^{\prime}, p, \theta^{\prime}\right) .
\end{aligned}
$$

Therefore, instead of 4 sets of 4 coupled equations for $T_{\lambda_{1}^{\prime} \lambda_{2}^{\prime} \lambda_{1} \lambda_{2}}^{a, \tau v}\left(p^{\prime}, p, \theta^{\prime}\right)$ given in Eq. (2.9), we need to solve only 2 sets of 3 coupled equations. One set is for $T_{\frac{1}{2} \frac{1}{2} \frac{1}{2} \frac{1}{2}}^{a, \tau v}\left(p^{\prime}, p, \theta^{\prime}\right), T_{\frac{1}{2},-\frac{1}{2}, \frac{1}{2} \frac{1}{2}}^{a, \tau v}\left(p^{\prime}, p, \theta^{\prime}\right)$, $T_{-\frac{1}{2},-\frac{1}{2}, \frac{1}{2} \frac{1}{2}}^{a, \tau v}\left(p^{\prime}, p, \theta^{\prime}\right)$ and the other for $T_{\frac{1}{2},-\frac{1}{2}, \frac{1}{2},-\frac{1}{2}}^{a, \tau v}\left(p^{\prime}, p, \theta^{\prime}\right), T_{\frac{1}{2} \frac{1}{2} \frac{1}{2},-\frac{1}{2}}^{a, \tau v^{2}}\left(p^{\prime}, p, \theta^{\prime}\right), T_{-\frac{1}{2}, \frac{1}{2} \frac{1}{2},-\frac{1}{2}}^{a, \tau v}\left(p^{\prime}, p, \theta^{\prime}\right)$.

With the xz plane being the scattering plane, this leads to

$$
T_{\lambda_{1}^{\prime} \lambda_{2}^{\prime} \lambda_{1} \lambda_{2}}^{a, \nu \nu}\left(p \hat{\mathbf{p}}^{\prime}, p \hat{\mathbf{z}}\right)=T_{\lambda_{1}^{\prime} \lambda_{2}^{\prime} \lambda_{1} \lambda_{2}}^{a, \tau \nu}\left(p, p, \theta^{\prime}\right) .
$$

Thus, as an example, the proton-proton spin-averaged differential cross section can be calculated from the 6 T-matrix elements of the type $T_{\lambda_{1}^{\prime} \lambda_{2}^{\prime} \lambda_{1} \lambda_{2}}^{a,{ }^{2}}\left(p^{\prime}, p, \theta^{\prime}\right)$ mentioned above as

$$
\begin{aligned}
\frac{\overline{d \sigma}}{d \hat{\mathbf{p}}^{\prime}}=\frac{1}{2} \mu^{2}(2 \pi)^{4} & \left\{\left|T_{\frac{1}{2} \frac{1}{2} \frac{1}{2} \frac{1}{2}}^{a, 11}\left(p, p, \theta^{\prime}\right)\right|^{2}+2\left|T_{\frac{1}{2},-\frac{1}{2} \frac{1}{2} \frac{1}{2}}^{a, 11}\left(p, p, \theta^{\prime}\right)\right|^{2}+\left|T_{-\frac{1}{2},-\frac{1}{2} \frac{1}{2} \frac{1}{2}}^{a, 11}\left(p, p, \theta^{\prime}\right)\right|^{2}\right. \\
& \left.+\left|T_{\frac{1}{2},-\frac{1}{2} \frac{1}{2},-\frac{1}{2}}^{a, 11}\left(p, p, \theta^{\prime}\right)\right|^{2}+2\left|T_{\frac{1}{2} \frac{1}{2} \frac{1}{2},-\frac{1}{2}}^{a, 11}\left(p, p, \theta^{\prime}\right)\right|^{2}+\left|T_{-\frac{1}{2} \frac{1}{2} \frac{1}{2},-\frac{1}{2}}^{a, 11}\left(p, p, \theta^{\prime}\right)\right|^{2}\right\} .
\end{aligned}
$$




\section{Spin operators for any NN interaction model}

A set of six spin operators is defined in Ref. [8] to describe any interaction between not only NN but any two spin-half particles. These spin operators are

$$
\begin{array}{ll}
P_{1}\left(\hat{\mathbf{p}}^{\prime}, \hat{\mathbf{p}}\right)=1 & P_{4}\left(\hat{\mathbf{p}}^{\prime}, \hat{\mathbf{p}}\right)=\left(\sigma_{1} \cdot \hat{\mathbf{p}}\right)\left(\sigma_{2} \cdot \hat{\mathbf{p}}\right) \\
P_{2}\left(\hat{\mathbf{p}}^{\prime}, \hat{\mathbf{p}}\right)=\sigma_{1} \cdot \sigma_{2} & P_{5}\left(\hat{\mathbf{p}}^{\prime}, \hat{\mathbf{p}}\right)=\left(\sigma_{1} \cdot \hat{\mathbf{p}}^{\prime}\right)\left(\sigma_{1} \cdot \hat{\mathbf{p}}\right)+\left(\sigma_{2} \cdot \hat{\mathbf{p}}^{\prime}\right)\left(\sigma_{2} \cdot \hat{\mathbf{p}}\right) . \\
P_{3}\left(\hat{\mathbf{p}}^{\prime}, \hat{\mathbf{p}}\right)=\left(\sigma_{1} \cdot \hat{\mathbf{p}}^{\prime}\right)\left(\sigma_{2} \cdot \hat{\mathbf{p}}^{\prime}\right) & P_{6}\left(\hat{\mathbf{p}}^{\prime}, \hat{\mathbf{p}}\right)=\left(\sigma_{1} \cdot \hat{\mathbf{p}}^{\prime}\right)\left(\sigma_{2} \cdot \hat{\mathbf{p}}\right)+\left(\sigma_{1} \cdot \hat{\mathbf{p}}\right)\left(\sigma_{2} \cdot \hat{\mathbf{p}}^{\prime}\right)
\end{array}
$$

Thus, we express any NN potential in terms of these six operators $P_{i}\left(\hat{\mathbf{p}}^{\prime}, \hat{\mathbf{p}}\right)$ as

$$
V^{\tau v}\left(\mathbf{p}^{\prime}, \mathbf{p}\right) \equiv\left\langle\tau v\left|\left\langle\mathbf{p}^{\prime}|V| \mathbf{p}\right\rangle\right| \tau v\right\rangle=\sum_{i=1}^{6} f_{i}^{\tau v}\left(p^{\prime}, p, \hat{\mathbf{p}}^{\prime} \cdot \hat{\mathbf{p}}\right) P_{i}\left(\hat{\mathbf{p}}^{\prime}, \hat{\mathbf{p}}\right)
$$

with $f_{i}^{\tau v}\left(p^{\prime}, p, \hat{\mathbf{p}}^{\prime} \cdot \hat{\mathbf{p}}\right)$ being some spin-independent functions.

Let us take a look at, for example, the Bonn realistic NN potential[9]. Some of the spin operators of the Bonn potential are already some of those operators $P_{i}\left(\hat{\mathbf{p}}^{\prime}, \hat{\mathbf{p}}\right)$. There are four spin operators of the Bonn potential, which are not one of the operators $P_{i}\left(\hat{\mathbf{p}}^{\prime}, \hat{\mathbf{p}}\right)$, but these four operators can be writen in terms of the operators $P_{i}\left(\hat{\mathbf{p}}^{\prime}, \hat{\mathbf{p}}\right)$ as shown in the following, with $\gamma=\hat{\mathbf{p}}^{\prime} \cdot \hat{\mathbf{p}}$ :

$$
\begin{aligned}
& \sigma_{1} \cdot \sigma_{2} \sigma_{1} \cdot \mathbf{p} \sigma_{2} \cdot \mathbf{p}=p^{2}\left\{P_{1}\left(\hat{\mathbf{p}}^{\prime}, \hat{\mathbf{p}}\right)-P_{2}\left(\hat{\mathbf{p}}^{\prime}, \hat{\mathbf{p}}\right)+P_{4}\left(\hat{\mathbf{p}}^{\prime}, \hat{\mathbf{p}}\right)\right\} \\
& \sigma_{1} \cdot \mathbf{p}^{\prime} \sigma_{2} \cdot \mathbf{p}^{\prime} \sigma_{1} \cdot \sigma_{2}=p^{\prime 2}\left\{P_{1}\left(\hat{\mathbf{p}}^{\prime}, \hat{\mathbf{p}}\right)-P_{2}\left(\hat{\mathbf{p}}^{\prime}, \hat{\mathbf{p}}\right)+P_{3}\left(\hat{\mathbf{p}}^{\prime}, \hat{\mathbf{p}}\right)\right\} \\
& \sigma_{1} \cdot \mathbf{p}^{\prime} \sigma_{2} \cdot \mathbf{p}^{\prime} \sigma_{1} \cdot \mathbf{p} \sigma_{2} \cdot \mathbf{p}=p^{\prime 2} p^{2}\left[-\gamma^{2} P_{1}\left(\hat{\mathbf{p}}^{\prime}, \hat{\mathbf{p}}\right)-\left(1-\gamma^{2}\right) P_{2}\left(\hat{\mathbf{p}}^{\prime}, \hat{\mathbf{p}}\right)+P_{3}\left(\hat{\mathbf{p}}^{\prime}, \hat{\mathbf{p}}\right)\right. \\
& \left.+P_{4}\left(\hat{\mathbf{p}}^{\prime}, \hat{\mathbf{p}}\right)+\gamma\left\{P_{5}\left(\hat{\mathbf{p}}^{\prime}, \hat{\mathbf{p}}\right)-P_{6}\left(\hat{\mathbf{p}}^{\prime}, \hat{\mathbf{p}}\right)\right\}\right] \\
& \sigma_{1} \cdot \mathbf{p}^{\prime} \sigma_{1} \cdot \sigma_{2} \sigma_{2} \cdot \mathbf{p}+\sigma_{2} \cdot \mathbf{p}^{\prime} \sigma_{1} \cdot \sigma_{2} \sigma_{1} \cdot \mathbf{p}=p^{\prime} p\left[-2 \gamma\left\{P_{1}\left(\hat{\mathbf{p}}^{\prime}, \hat{\mathbf{p}}\right)-P_{2}\left(\hat{\mathbf{p}}^{\prime}, \hat{\mathbf{p}}\right)\right\}\right. \\
& \left.-P_{6}\left(\hat{\mathbf{p}}^{\prime}, \hat{\mathbf{p}}\right)+2 P_{5}\left(\hat{\mathbf{p}}^{\prime}, \hat{\mathbf{p}}\right)\right] \text {. }
\end{aligned}
$$

The spin matrix elements $P_{\lambda_{1}^{\prime} \lambda_{2}^{\prime} \lambda_{1} \lambda_{2}}^{(i)}\left(\hat{\mathbf{p}}^{\prime}, \hat{\mathbf{p}}\right) \equiv\left\langle\hat{\mathbf{z}} \lambda_{1}^{\prime}\left|\left\langle\hat{\mathbf{z}} \lambda_{2}^{\prime}\left|P_{i}\left(\hat{\mathbf{p}}^{\prime}, \hat{\mathbf{p}}\right)\right| \hat{\mathbf{z}} \lambda_{1}\right\rangle\right| \hat{\mathbf{z}} \lambda_{2}\right\rangle$ result as

$$
\begin{aligned}
P_{\lambda_{1}^{\prime} \lambda_{2}^{\prime} \lambda_{1} \lambda_{2}}^{(1)}\left(\hat{\mathbf{p}}^{\prime}, \hat{\mathbf{p}}\right)= & \delta_{\lambda_{1}^{\prime} \lambda_{1}} \delta_{\lambda_{2}^{\prime} \lambda_{2}} \\
P_{\lambda_{1}^{\prime} \lambda_{2}^{\prime} \lambda_{1} \lambda_{2}}^{(2)}\left(\hat{\mathbf{p}}^{\prime}, \hat{\mathbf{p}}\right)= & \delta_{\lambda_{1}^{\prime} \lambda_{1}}\left(\delta_{\lambda_{1}^{\prime} \lambda_{2}^{\prime}} \delta_{\lambda_{1} \lambda_{2}}-\delta_{\lambda_{1}^{\prime},-\lambda_{2}^{\prime}} \delta_{\lambda_{1},-\lambda_{2}}\right)+2 \delta_{\lambda_{1}^{\prime},-\lambda_{1}} \delta_{\lambda_{1}^{\prime},-\lambda_{2}^{\prime}} \delta_{\lambda_{1},-\lambda_{2}} \\
P_{\lambda_{1}^{\prime} \lambda_{2}^{\prime} \lambda_{1} \lambda_{2}}^{(3)}\left(\hat{\mathbf{p}}^{\prime}, \hat{\mathbf{p}}\right)= & \left(\delta_{\lambda_{1}^{\prime} \lambda_{1}} 2 \lambda_{1} \cos \theta^{\prime}+\delta_{\lambda_{1}^{\prime},-\lambda_{1}} e^{2 i \lambda_{1} \phi^{\prime}} \sin \theta^{\prime}\right) \\
& \times\left(\delta_{\lambda_{2}^{\prime} \lambda_{2}} 2 \lambda_{2} \cos \theta^{\prime}+\delta_{\lambda_{2}^{\prime},-\lambda_{2}} e^{2 i \lambda_{2} \phi^{\prime}} \sin \theta^{\prime}\right) \\
P_{\lambda_{1}^{\prime} \lambda_{2}^{\prime} \lambda_{1} \lambda_{2}}^{(4)}\left(\hat{\mathbf{p}}^{\prime}, \hat{\mathbf{p}}\right)= & \left(\delta_{\lambda_{1}^{\prime} \lambda_{1}} 2 \lambda_{1} \cos \theta+\delta_{\lambda_{1}^{\prime},-\lambda_{1}} e^{2 i \lambda_{1} \phi} \sin \theta\right) \\
& \times\left(\delta_{\lambda_{2}^{\prime} \lambda_{2}} 2 \lambda_{2} \cos \theta+\delta_{\lambda_{2}^{\prime},-\lambda_{2}} e^{2 i \lambda_{2} \phi} \sin \theta\right) \\
P_{\lambda_{1}^{\prime} \lambda_{2}^{\prime} \lambda_{1} \lambda_{2}}^{(5)}\left(\hat{\mathbf{p}}^{\prime}, \hat{\mathbf{p}}\right)= & \delta_{\lambda_{2}^{\prime} \lambda_{2}}\left[\delta_{\lambda_{1}^{\prime} \lambda_{1}}\left\{\cos \theta^{\prime} \cos \theta+e^{-2 i \lambda_{1}\left(\phi^{\prime}-\phi\right)} \sin \theta^{\prime} \sin \theta\right\}\right. \\
& \left.+\delta_{\lambda_{1}^{\prime},-\lambda_{1}} 2 \lambda_{1} e^{2 i \lambda_{1} \phi^{\prime}}\left\{\sin \theta^{\prime} \cos \theta-e^{-2 i \lambda_{1}\left(\phi^{\prime}-\phi\right)} \cos \theta^{\prime} \sin \theta\right\}\right] \\
& +\delta_{\lambda_{1}^{\prime} \lambda_{1}}\left[\delta_{\lambda_{2}^{\prime} \lambda_{2}}\left\{\cos \theta^{\prime} \cos \theta+e^{-2 i \lambda_{2}\left(\phi^{\prime}-\phi\right)} \sin \theta^{\prime} \sin \theta\right\}\right.
\end{aligned}
$$




$$
\begin{aligned}
& \left.+\delta_{\lambda_{2}^{\prime},-\lambda_{2}} 2 \lambda_{2} e^{2 i \lambda_{2} \phi^{\prime}}\left\{\sin \theta^{\prime} \cos \theta-e^{-2 i \lambda_{2}\left(\phi^{\prime}-\phi\right)} \cos \theta^{\prime} \sin \theta\right\}\right] \\
P_{\lambda_{1}^{\prime} \lambda_{2}^{\prime} \lambda_{1} \lambda_{2}}^{(6)}\left(\hat{\mathbf{p}}^{\prime}, \hat{\mathbf{p}}\right)= & \left(\delta_{\lambda_{1}^{\prime} \lambda_{1}} 2 \lambda_{1} \cos \theta^{\prime}+\delta_{\lambda_{1}^{\prime},-\lambda_{1}} e^{2 i \lambda_{1} \phi^{\prime}} \sin \theta^{\prime}\right) \\
& \times\left(\delta_{\lambda_{2}^{\prime} \lambda_{2}} 2 \lambda_{2} \cos \theta+\delta_{\lambda_{2}^{\prime},-\lambda_{2}} e^{2 i \lambda_{2} \phi} \sin \theta\right) \\
& +\left(\delta_{\lambda_{1}^{\prime} \lambda_{1}} 2 \lambda_{1} \cos \theta+\delta_{\lambda_{1}^{\prime},-\lambda_{1}} e^{2 i \lambda_{1} \phi} \sin \theta\right) \\
& \times\left(\delta_{\lambda_{2}^{\prime} \lambda_{2}} 2 \lambda_{2} \cos \theta^{\prime}+\delta_{\lambda_{2}^{\prime},-\lambda_{2}} e^{2 i \lambda_{2} \phi^{\prime}} \sin \theta^{\prime}\right) .
\end{aligned}
$$

Due to the Kronecker delta's in Eqs. (3.7) - (3.12) many of the spin matrix elements $P_{\lambda_{1}^{\prime} \lambda_{2}^{\prime} \lambda_{1} \lambda_{2}}^{(i)}\left(\hat{\mathbf{p}}^{\prime}, \hat{\mathbf{p}}\right)$ vanish. In the following we show some of the nonvanishing ones, with $\lambda= \pm \frac{1}{2}$ :

$P_{\lambda \lambda \lambda \lambda}^{(1)}\left(\hat{\mathbf{p}}^{\prime}, \hat{\mathbf{p}}\right)=1 \quad P_{\lambda \lambda,-\lambda,-\lambda}^{(4)}\left(\hat{\mathbf{p}}^{\prime}, \hat{\mathbf{p}}\right)=e^{-4 i \lambda \phi} \sin ^{2} \theta$

$P_{\lambda \lambda \lambda \lambda}^{(2)}\left(\hat{\mathbf{p}}^{\prime}, \hat{\mathbf{p}}\right)=1 \quad P_{\lambda \lambda \lambda \lambda}^{(5)}\left(\hat{\mathbf{p}}^{\prime}, \hat{\mathbf{p}}\right)=2\left\{\cos \theta^{\prime} \cos \theta+e^{-2 i \lambda\left(\phi^{\prime}-\phi\right)} \sin \theta^{\prime} \sin \theta\right\}$.

$P_{\lambda \lambda,-\lambda,-\lambda}^{(3)}\left(\hat{\mathbf{p}}^{\prime}, \hat{\mathbf{p}}\right)=e^{-4 i \lambda \phi^{\prime}} \sin ^{2} \theta^{\prime} \quad P_{\lambda \lambda \lambda \lambda}^{(6)}\left(\hat{\mathbf{p}}^{\prime}, \hat{\mathbf{p}}\right)=2 \cos \theta^{\prime} \cos \theta$

\section{Summary}

Three-dimensional calculations are shown to be necessary or even required, especially for NN and $3 \mathrm{~N}$ scattering at higher energies of a few hundreds MeV. As NN T-matrix elements are input to $3 \mathrm{~N}$ scattering calculations, we develope a new 3D formulation starting with $\mathrm{NN}$ scattering. We derive integral equations for NN T-matrix elements in new 3D basis. The final integral equations consist of 2 sets of 3 coupled equations for the T-matrix elements in two variables, i.e. the magnitude of the relative momentum and the scattering angle. The solution of these equations can be used directly to calculate observables of NN scattering. This is shown, as an example, for the spinaveraged differential cross section. We evaluate matrix elements of six spin operators, in terms of which any NN potential can be discribed, as an example, the Bonn potential.

\section{References}

[1] I. Fachruddin, Ch. Elster and W. Glöckle, Nucl. Phys. A689, 507c (2001).

[2] J. Golak, et.al., Phys. Rev. C81, 034006 (2010).

[3] I. Fachruddin, Ch. Elster and W. Glöckle, Phys. Rev. C68, 054003 (2003).

[4] Ch. Elster, J. H. Thomas, W. Glöckle, Few-Body Systems 24, 55 (1998).

[5] W. Schadow, Ch. Elster, W. Glöckle, Few-Body Systems 28, 15 (2000).

[6] I. Fachruddin, Ch. Elster and W. Glöckle, Phys. Rev. C62, 044002 (2000).

[7] W. Glöckle, et.al., Eur. Phys. J. A43, 339 (2010).

[8] I. Fachruddin, Few-Body Syst. 54, 1621 (2013), DOI 10.1007/s00601-012-0513-0.

[9] R. Machleidt, K. Holinde, Ch. Elster, Phys. Rep. 149, 1 (1987). 